\title{
The Effect of Glutamine Supplementation on Oxidative Stress and Matrix Metalloproteinase 2 and 9 After Exhaustive Exercise
}

This article was published in the following Dove Press journal:

Drug Design, Development and Therapy

\author{
Ali Nemati $\mathbb{D}^{\prime}$ \\ Reza Alipanah- \\ Moghadam (iD)' \\ Leila Molazadeh' \\ Abbas Naghizadeh Baghi (D) ${ }^{2}$ \\ 'Ardabil University of Medical Sciences, \\ School of Medicine, Department of \\ Clinical Biochemistry, Ardabil, Iran; \\ ${ }^{2}$ University of Mohaghegh Ardabili, \\ Department of Exercise and Physiology, \\ Ardabil, Iran
}

Background: Glutamine is the most abundant amino acid in plasma and skeletal muscles and an important fuel for immune system cells. It has beneficial anti-inflammatory and antioxidant properties which may be considered as a potentially useful supplement for athletes. The present study was conducted to investigate the effect of glutamine supplementation on oxidative stress and matrix metalloproteinase 2 and 9 after exhaustive exercise in young healthy males.

Materials and methods: In this study, 30 healthy males (supplement $=15$ and control=15) were randomly assigned into two groups. The supplement group received $0.3 \mathrm{~g} / \mathrm{kg} \mathrm{BW}$ of glutamine along with $25 \mathrm{gr}$ of sugar dissolved in $250 \mathrm{cc}$ water per day. The control group received $25 \mathrm{gr}$ of sugar in $250 \mathrm{cc}$ water per day. Fasting blood samples were taken at baseline and at the end of 14 days of intervention. The participants underwent exercise until experiencing fullbody exhaustive fatigue for $16 \pm 2.84 \mathrm{mins}$, and then fasting blood samples were taken. Serum levels of TAC, MDA, MMP2, MMP9, glutathione, and hs-CRP were measured.

Results: Serum levels of MDA and hs-CRP significantly decreased in the supplement group $(p<0.05)$. The serum level of TAC significantly increased in the supplement group $(p<0.05)$. Glutathione serum levels significantly increased after exhaustive exercise $(p<0.05)$. Serum levels of MMP2 and MMP9 remained unchanged.

Conclusion: Results of this study showed that, some biochemical factors are timedependent and can increase or decrease over time, as well as, serum levels of hs-CRP and MDA decreased with glutamine supplementation along with the increase in the TAC serum levels, but this supplementation had no effect on serum levels of MMP2 and MMP9 in exhaustive exercise.

Keywords: glutamine, oxidative stress, exhaustive exercise, MMP2\&9

\section{Introduction}

Physical activity has many health benefits, but it can result in the production of molecules of free radicals and damage to cells. ${ }^{1}$ In intense physical activity, enhanced oxygen consumption and increased Reactive Oxygen Species (ROS) production may damage the cells. ${ }^{2}$ Moreover, high-intensity exercise may stimulate the immune system leading to an increase in the levels of inflammatory biomarkers including high-sensitivity C-reactive protein (hs-CRP) and matrix metalloproteinases (MMPs) such as MMP2 and MMP9. ${ }^{3}$ MMPs are zinc-dependent endopeptidases involved in the degradation of extracellular proteoglycans and breakdown of matrix proteins. ${ }^{4,5}$ Local production of matrix metalloproteinases in skeletal
Correspondence: Reza Alipanah-Moghadam Tel +98914157II67

Email alipanahreza9@gmail.com 
muscles has been observed at high levels of mechanical stress such as eccentric or high-impact exercise. ${ }^{6}$ Also, some studies have reported that serum levels of MMP2 and MMP9 increase after short-duration exhaustive exercises. ${ }^{7}$ It has been reported that the MMP2 and MMP9 may play an important role in inflammatory myopathies in skeletal muscles. ${ }^{8}$ Furthermore, regular consumption of natural antioxidants has been shown to reduce ROS production and it may modulate inflammation in exhaustive exercise. ${ }^{9,10}$ Glutamine is the most abundant amino acid in the human body contributing to glutathione production, as a part of the antioxidant system, and also has anti-inflammatory effects. ${ }^{11}$ It has been shown that in exhaustive exercise, glutamine depletion occurs, which can be accompanied by tissue damage. Thus, the consumption of glutamine supplements may be beneficial for athletes. $^{12}$ Although a number of studies have demonstrated possible benefits of glutamine consumption in animal and human subjects, ${ }^{13,14}$ there is not enough evidence to establish positive effects of glutamine supplementation on inflammatory factors as well as MMP2 and MMP9 and oxidative stress in relation to exhaustive exercise. ${ }^{15}$ Regarding antioxidant and anti-inflammatory properties of glutamine, this study is assigned to investigate the effect of glutamine supplementation on oxidative stress and MMP2 and MMP9 after an exhaustive exercise in young healthy males.

\section{Materials and Methods Study Design}

This study was conducted at Ardabil University of Medical Sciences, Ardabil, Iran, that was approved by the Institutional Ethics Committee with the ethical code of IR.ARUMS.REC.1394.112 and was recorded in the Iranian Registry of Clinical Trials with the identification code of IRCT2016092129895N1 and it was conducted in accordance with the Declaration of Helsinki. Forty healthy individuals were recruited at the beginning of the study. Ten individuals were excluded from the study due to lack of cooperation and abuse of glutamine supplements. Thirty healthy participants were selected and divided into two groups (control group=15 and supplement group=15). The sample size for each group included 15 individuals using either the formula of: $n=\left[\left(Z_{1-\alpha / 2}\right)+\left(Z_{1-\beta}\right)\right]^{2} \times\left(\delta_{1}{ }^{2}+\right.$ $\left.\delta_{2}{ }^{2}\right):\left(\mu_{2}-\mu_{1}\right)^{2}$ or the PASS11 software. ${ }^{16}$ Participants with heart, lung, kidney and other organ diseases, smokers, alcohol consumers and those who follow a specific kind of eating behavior (such as eating for losing or gaining weight), and consumers of other supplements like vitamins and antioxidants were excluded from the study.

\section{Participants}

The participants who volunteered for this study were 30 healthy adult males with moderate physical activity, normal weight, and Body Mass Index (BMI) range of 18.5-25 Kg/m within the age range of 18-30 years old, who were randomly assigned into two groups. Physical activity criteria of the participants were having a moderate activity such as cycling, fast walking, jogging, running, dancing, and swimming. Purpose of the investigation and sport discipline such as fasting, running on a treadmill until exhaustion, blood sampling were described to the participants, and a written consent was obtained from all the participants before filling the questionnaire. All the participants completed the general questionnaire, and information on anthropometric factors (height and weight) was collected at the beginning of the study. Also, explanations on optimal conditions for cooperation, as well as how to fill out the $24 \mathrm{hr}$ recall questionnaire were given to the participants in a question -and -answer session. Participants were prohibited from taking any supplements, anti-inflammatory drugs, and performing intensive exercise for 3 days before the start of the study. After obtaining the written consent form, recording general information, assessing anthropometric factors (height and weight), and also taking fasting blood samples, a dose of 0.3 gr of glutamine per kilogram of body weight per day with 25 gr of sugar dissolved in $250 \mathrm{CC}$ water was given to the supplement group for 14 days. On the 15 th day, a plan of an exhaustive exercise was given for both groups. Dosage and time of glutamine supplementation were selected based on previous studies. ${ }^{17,18}$ The control group received only $25 \mathrm{gr}$ of sugar dissolved in $250 \mathrm{CC}$ water per day for 14 days. Fasting blood samples were taken from all the participants at the end of 14 days of intervention and immediately after the exhausting exercise by venipuncture, and the sera were separated. The collected sera were kept frozen at $-80{ }^{\circ} \mathrm{C}$ till analysis.

\section{Exhaustive Exercise}

The participants fasted for 10-12 hrs prior to the test, and then were given a plan of an exhaustive exercise. At first, all the participants were warmed up and trained under the supervision of a physical education instructor for 5 to 10 mins. Afterward, the participants were given a plan of an exhaustive exercise according to the Bruce test until experiencing fatigue for $16 \pm 2.84 \mathrm{mins}$, and then blood samples 
were immediately taken. Bruce test is a standard maximal aerobic test in which the subject for 3 mins at a speed of $2.74 \mathrm{~km} / \mathrm{h}$ with $10 \%$ slope begins to walk on the treadmill, secondly, at a speed of $4.02 \mathrm{~km} / \mathrm{h}$ with $12 \%$ slope moves on the treadmill for 3 mins, and the next steps involve adding treadmill speed and adding up to $2 \%$ slope for 3 mins. ${ }^{19,20}$ Table 1 shows the slope and speed of the treadmill during exhaustive exercise according to the Bruce test. The length of time spent on the treadmill is considered as the test score and can be used to estimate the VO2 max value. VO2 max is the maximum oxygen utilizing during intense exercise. ${ }^{21}$ An estimate of the athlete's VO2 max can be calculated by the Pollock's formula (VO2 $\max =4.38 \times \mathrm{T}-3.9$ ) based on the total run time. ${ }^{22}$

\section{Biochemical Information}

MMP2 (EH0017) and MMP9 (EH0238) serum levels were assayed by the standard Enzyme-Linked Immunosorbent Assay (ELISA) kits with intra- and inter-assay CVs less than $8 \%$ and $10 \%$, respectively. Kit detection ranges in MMP 2 and MMP9 ELISA kits were 0.5-32 and 0.313-20 ng/mL, respectively. Serum samples were diluted at a ratio of 1:100 according to the manufacturer's specifications for MMP2 and MMP9 assay. Serum of Total Antioxidant Capacity (TAC) was measured by the colorimetric method using a standard kit (NX2332). Kit detection range for TAC was up to 2.50 $\mathrm{mmol} / \mathrm{l}$. Malondialdehyde (MDA) serum levels were measured through the Thiobarbituric Acid Reactive Substances (TBARS) method. Samples were heated with $0.6 \%$ thiobarbituric acid (T5500) under acidic condition and after cooling, the colored product was extracted into n-butanol. The pink color absorbance was measured at $530 \mathrm{~nm}$. The MDA standards were prepared using 1, 1, 3, 3-tetraethoxypropane (T988925ML). Immunoturbidometric method was used for measurement of hs-CRP with intra- and inter-assay CV less than $2 \%$. Kit detection range for hs-CRP was $0.1-20 \mathrm{mg} / \mathrm{l}$. Glutathione

Table I The Slope and Speed of Treadmill During Exhaustive Exercise

\begin{tabular}{|l|l|l|l|}
\hline Steps & Time (min) & Speed (km/h) & Slope (\%) \\
\hline 1 & 0 & 2.74 & $10 \%$ \\
2 & 3 & 4.02 & $12 \%$ \\
3 & 6 & 5.47 & $14 \%$ \\
4 & 9 & 6.76 & $16 \%$ \\
5 & 12 & 8.05 & $18 \%$ \\
6 & 15 & 8.85 & $20 \%$ \\
7 & 18 & 9.65 & $22 \%$ \\
\hline
\end{tabular}

serum levels were measured by standard ELISA kit with intraand inter-assay CVs less than $4 \%$ and $5 \%$, respectively (ZBGSH-96A). Kit detection range for glutathione was 0.03-1 mM.

\section{Nutritional Information}

Food intake was recorded using 24 hrs dietary recalls for 3 days ( 2 weekdays and 1 weekend day) during the study. The content of the nutrients (macronutrients and micronutrients) and the energy intake of the participants were measured and analyzed by the Nutritionist software IV.

\section{Data Analysis}

Data were analyzed using the Shapiro-Wilk tests, Independent-Samples T-test and Paired-Samples T-test. Also, the Repeated Measures Analysis of Variance was used to evaluate changes of biochemical parameters within the groups at different times with a significant level. Statistical analysis was performed using SPSS software 22. This study was conducted with a confidence level of $95 \%$ and a test power of $80 \%$. For all tests, a P-value of $<0.05$ was considered as statistically significant.

\section{Results}

The average age of the participants in the supplement and control groups was equal to $19.66 \pm 2.02$ vs $18.73 \pm 0.7$ years old, respectively. The duration of training time was higher in the supplement group than the control group (17.45 \pm 2.79 vs $14.71 \pm 2.23 \mathrm{~min}$.) $(\mathrm{p}<0.05)$. Although the mean of VO2 max was more in the supplement group than the control group $(50.77 \pm 9.52$ vs $44.46 \pm 8.76 \mathrm{~mL} / \mathrm{kg} / \mathrm{min})$, but this difference was not significant $(\mathrm{p}=0.313)$. Information on anthropometric factors and nutritional intake in the control and supplement groups and their comparison between the two groups are presented in Table 2. Results of the Independent-Samples $T$-test showed that there was no significant difference regarding the variables (such as weight, BMI, calorie, and nutrients intake) between the two groups. Calories and nutrients consumed by both groups were the same. Glutamine supplementation had no significant effect on serum levels of MMP2 and MMP9 and no significant difference was observed in serum levels of MMP2 and MMP9 between the two groups at the beginning and end of the study (Table 3 ). Serum levels of MMP2 and MMP9 increased and decreased insignificantly after exhaustive exercise in the supplement and control groups, respectively. Results showed no significant difference in the serum level of 
Table 2 The Comparison of Anthropometric Factors and Nutritional Intake in Control and Supplement Groups

\begin{tabular}{|c|c|c|c|c|c|c|c|}
\hline \multirow[t]{2}{*}{ Variables } & \multicolumn{2}{|l|}{ Groups } & \multirow[t]{2}{*}{$P$ value } & \multirow[t]{2}{*}{ Variables } & \multicolumn{2}{|l|}{ Groups } & \multirow[t]{2}{*}{$P$ value } \\
\hline & Supplement M $\pm S D$ & Control M $\pm S D$ & & & Supplement M $\pm S D$ & Control M $\pm S D$ & \\
\hline Weight (kg) & $72.32 \pm 12.6$ & $79.27 \pm 13.58$ & 0.16 & V. $B_{I}(m g)$ & $1.5 \pm 0.21$ & $1.55 \pm 0.38$ & 0.65 \\
\hline BMI $\left(\mathrm{kg} / \mathrm{m}^{2}\right)$ & $22.06 \pm 3.68$ & $24.15 \pm 3.92$ & 0.14 & V. $B_{2}(m g)$ & $1.33 \pm 0.27$ & $1.39 \pm 0.35$ & 0.62 \\
\hline Height (cm) & $|8| .06 \pm 4.3 \mid$ & $180.66 \pm 2.16$ & 0.8 & V. $B_{3}(m g)$ & $24.43 \pm 8.11$ & $23.75 \pm 3.81$ & 0.77 \\
\hline Cal (Kcal/day) & $2463.5 \pm 255.5$ & $2553 \pm 386.7$ & 0.46 & V. $B_{6}(m g)$ & $1.35 \pm 0.46$ & $1.1 \pm 0.29$ & 0.06 \\
\hline Protein $(g)$ & $95.6 \pm 13.1$ & $95.33 \pm 13.3$ & 0.96 & V. $B_{9}(\mu g)$ & $121.2 \pm 70.2$ & $118.5 \pm 47.7$ & 0.9 \\
\hline $\mathrm{CHO}(\mathrm{g})$ & $327.3 \pm 32.2$ & $346.3 \pm 70.5$ & 0.35 & V. C (mg) & $51 \pm 35.28$ & $56.7 \pm 24.7$ & 0.61 \\
\hline Total fat $(g)$ & $85.2 \pm 12.1$ & $87.4 \pm 15.2$ & 0.66 & $\mathrm{Ca}(\mathrm{mg})$ & $609.07 \pm 221.74$ & $634.53 \pm 273.6$ & 0.78 \\
\hline $\mathrm{SF}(\mathrm{g})$ & $16.13 \pm 2.9$ & $19.13 \pm 5.8$ & 0.09 & $\mathrm{Fe}(\mathrm{mg})$ & $20.8 \pm 5.47$ & $24.2 \pm 7.26$ & 0.16 \\
\hline PUFA (g) & $13.4 \pm 4.7$ & $13.6 \pm 4.1$ & 0.9 & $\mathrm{Zn}(\mathrm{mg})$ & $4.19 \pm 1.04$ & $3.41 \pm 1.14$ & 0.06 \\
\hline MUFA (g) & $20.2 \pm 3.4$ & $22.9 \pm 5.5$ & 0.12 & $\mathrm{Cu}(\mathrm{mg})$ & $0.93 \pm 0.26$ & $0.86 \pm 0.14$ & 0.36 \\
\hline Glutamic Acid (g) & $3.9 \pm 1.7$ & $3.3 \pm 1.7$ & 0.39 & $M g(m g)$ & $153.7 \pm 58.38$ & $143.2 \pm 34.52$ & 0.56 \\
\hline Glucose (g) & $4.2 \pm 3.9$ & $3.1 \pm 2.8$ & 0.32 & $\mathrm{~K}(\mathrm{mg})$ & $2013.8 \pm 650.3$ & $1835.4 \pm 282.9$ & 0.34 \\
\hline Fructose (g) & $4.3 \pm 3.9$ & $3.6 \pm 3.1$ & 0.39 & Biotin (mg) & $10.9 \pm 10.4$ & $10.25 \pm 9.65$ & 0.97 \\
\hline V. E (mg) & $14 \pm 3.1$ & $15.4 \pm 2.9$ & 0.21 & V. $B_{12}(\mu g)$ & $3.8 \pm 1.29$ & $4.25 \pm 1.4$ & 0.37 \\
\hline V.D $(\mu g)$ & $1.26 \pm 0.93$ & $1.5 \pm 1.27$ & 0.25 & V.K $(\mu g)$ & $40.24 \pm 26.8$ & $52.33 \pm 49.7$ & 0.41 \\
\hline
\end{tabular}

Abbreviations: CHO, carbohydrate; BMI, Body Mass Index; V, Vitamine; V.Bg, Folic acid; Ca, Calcium; Mg, Magnesium; Cal, Calorie; MUFA, Mono unsaturated fat (g); PUFA, Poly unsaturated fat; SF, Saturated fat; K, Potassium; P, Phosphorous; Se, Selenium; Fe, Iron; Cu, Copper; Zn, Zinc.

Table 3 The Comparison of Matrix Metalloproteinase, Inflammatory and Oxidant Parameters Before and After 2 Weeks Glutamine Supplementation in Both Groups

\begin{tabular}{|c|c|c|c|c|}
\hline Variables & Measurement Stage & Supplement Group & Control Group & $P$ value \\
\hline \multirow[t]{2}{*}{ MMP2 (ng/mL) } & Before the intervention & $38.92 \pm 6.4$ & $35.18 \pm 8.35$ & 0.18 \\
\hline & Two weeks after the intervention & $35.46 \pm 7.79$ & $32.6 \pm 6.76$ & 0.29 \\
\hline$P$ value & & 0.06 & 0.2 & \\
\hline \multirow[t]{2}{*}{ MMP9 (ng/mL) } & Before the intervention & $924.55 \pm 362.58$ & $804.6 \pm 379.45$ & 0.38 \\
\hline & Two weeks after the intervention & $981.85 \pm 288.11$ & $988.36 \pm 417.34$ & 0.96 \\
\hline$P$ value & & 0.5 & 0.3 & \\
\hline \multirow[t]{2}{*}{ TAC (mmol/l) } & Before the intervention & $0.21 \pm 0.02$ & $0.22 \pm 0.02$ & 0.69 \\
\hline & Two weeks after the intervention & $0.23 \pm 0.02$ & $0.22 \pm 0.02$ & 0.83 \\
\hline$P$ value & & $0.03 *$ & 0.2 & \\
\hline \multirow[t]{2}{*}{ hs-CRP (mg/l) } & Before the intervention & $\mathrm{I} . \mathrm{I} \pm 0.94$ & I. $26 \pm 0.89$ & 0.65 \\
\hline & Two weeks after the intervention & $\mathrm{I} . \mathrm{I} \pm 0.7 \mathrm{I}$ & $1.92 \pm 1.83$ & $0.03^{\circ}$ \\
\hline$P$ value & & 0.9 & 0.1 & \\
\hline \multirow[t]{2}{*}{ MDA (nmol/l) } & Before the intervention & $12.15 \pm 3.32$ & $18.86 \pm 15.97$ & 0.12 \\
\hline & Two weeks after the intervention & $8.01 \pm 2.95$ & $18.07 \pm 13.83$ & $0.01^{\bullet}$ \\
\hline$P$ value & & $0.01 *$ & 0.8 & \\
\hline \multirow[t]{2}{*}{ Glutathione $(\mathrm{GSH})(\mu \mathrm{mol} / \mathrm{l})$} & Before the intervention & $16.06 \pm 8.19$ & $14.34 \pm 9.4$ & 0.59 \\
\hline & Two weeks after the intervention & $17.05 \pm 4.59$ & $19.73 \pm 7.77$ & 0.26 \\
\hline$P$ value & & 0.5 & 0.09 & \\
\hline
\end{tabular}

Notes: Values are Mean \pm SD. $* \mathrm{P}<0.05$ in paired-sample $t$-test vs baseline. ${ }^{\bullet} \mathrm{P}<0.05$ in independent-sample $t$-test between two groups

TAC at the beginning and end of supplementation between the two groups. However, the results of the PairedSamples $T$-test revealed that serum level of TAC increased significantly after 2 weeks of supplementation in the supplement group $(\mathrm{P}=0.003)$. Furthermore, the results of the Independent-Samples $T$-test revealed that serum level of 
TAC increased significantly after an exhaustive exercise in the supplement group compared to the control group $(\mathrm{P}=0.01)$. Statistical analysis of $t$-test showed that, at the end of 2 weeks of glutamine supplementation, serum levels of MDA and hs-CRP decreased significantly in the glutamine supplement group compared to the control group $(p<0.05)$. Results of the present study revealed that serum levels of MDA and hs-CRP decreased significantly after an exhaustive exercise in the glutamine supplement group compared to the control group $(\mathrm{p}<0.05)$. Also, results showed that glutathione serum levels significantly increased after exhaustive exercise $(\mathrm{P}=0.002)$ (Table 4). Results of the Repeated Measures Analysis of Variance showed a significant difference in variations in the biochemical values of TAC and MDA in the supplement group compared to the control group $(\mathrm{p}<0.05)$, and also these factors changed significantly over time in the supplement group but not in the control group. However, other biochemical factors in both groups showed the same pattern changes over time.

\section{Discussion}

This study was conducted to investigate the effect of glutamine supplementation as a nutritional supplement with antioxidant and anti-inflammatory properties after performing the exhaustive exercise. Results of our study showed that supplementation of glutamine with doses of $0.3 \mathrm{~g} / \mathrm{kg}$ of body weight per day for 14 days can reduce oxidative stress and improve the body's antioxidant system after supplementation and after an exhaustive exercise, but did not influence serum levels of MMP2 and MMP9. Previous studies have shown that intense exercise can be followed by oxidative stress and inflammation. ${ }^{23,24}$ Therefore, supplementation with compounds containing antioxidant and anti-inflammatory properties can be effective in improving this stress response against intense exercise. ${ }^{25}$ Recently, extensive scientific attention has been paid to glutamine, as the nonessential amino acid because of its antioxidant and anti-inflammatory effects in humans. ${ }^{13,26}$ Antioxidant effects of glutamine and its effects on improving antioxidant capability have been demonstrated in several studies. ${ }^{27,28}$ Antioxidant effects of glutamine can be exerted through

Table 4 The Comparison of Matrix Metalloproteinase, Inflammatory and Oxidant Parameters Before and After Exhaustive Exercise in Both Groups

\begin{tabular}{|c|c|c|c|c|}
\hline Variables & Measurement Stage & Supplement Group & Control Group & $P$ value \\
\hline MMP2 (ng/mL) & $\begin{array}{l}\text { Before exhaustive exercise } \\
\text { After exhaustive exercise }\end{array}$ & $\begin{array}{l}35.46 \pm 7.79 \\
36.49 \pm 7.75\end{array}$ & $\begin{array}{l}32.6 \pm 6.76 \\
35.03 \pm 8.22\end{array}$ & $\begin{array}{l}0.29 \\
0.62\end{array}$ \\
\hline$P$ value & & 0.16 & 0.9 & \\
\hline MMP9 (ng/mL) & $\begin{array}{l}\text { Before exhaustive exercise } \\
\text { After exhaustive exercise }\end{array}$ & $\begin{array}{l}981.85 \pm 288.11 \\
913.58 \pm 202.46\end{array}$ & $\begin{array}{l}988.36 \pm 417.34 \\
908.96 \pm 412.06\end{array}$ & $\begin{array}{l}0.96 \\
0.97\end{array}$ \\
\hline$P$ value & & 0.9 & 0.7 & \\
\hline TAC (mmol/l) & $\begin{array}{l}\text { Before exhaustive exercise } \\
\text { After exhaustive exercise }\end{array}$ & $\begin{array}{l}0.23 \pm 0.02 \\
0.24 \pm 0.02\end{array}$ & $\begin{array}{l}0.22 \pm 0.02 \\
0.22 \pm 0.01\end{array}$ & $\begin{array}{l}0.83 \\
0.01^{\bullet}\end{array}$ \\
\hline$P$ value & & $0.01 *$ & 0.34 & \\
\hline hs-CRP (mg/l) & $\begin{array}{l}\text { Before exhaustive exercise } \\
\text { After exhaustive exercise }\end{array}$ & $\begin{array}{l}\mathrm{I} . \mathrm{I} \pm 0.7 \mathrm{I} \\
\mathrm{I} .88 \pm 0.8\end{array}$ & $\begin{array}{l}1.92 \pm 1.83 \\
2.5 \pm 0.66\end{array}$ & $\begin{array}{l}0.03^{\bullet} \\
0.02^{\bullet}\end{array}$ \\
\hline$P$ value & & $0.03 *$ & $0.02 *$ & \\
\hline MDA (nmol/l) & $\begin{array}{l}\text { Before exhaustive exercise } \\
\text { After exhaustive exercise }\end{array}$ & $\begin{array}{l}8.01 \pm 2.95 \\
19.32 \pm 8.49\end{array}$ & $\begin{array}{l}18.07 \pm 13.83 \\
30.5 \pm 19.06\end{array}$ & $\begin{array}{l}0.01^{\bullet} \\
0.04^{\bullet}\end{array}$ \\
\hline$P$ value & & $0.02 *$ & 0.09 & \\
\hline Glutathione $(\mathrm{GSH})(\mu \mathrm{mol} / \mathrm{l})$ & $\begin{array}{l}\text { Before exhaustive exercise } 3 \\
\text { After exhaustive exercise }\end{array}$ & $\begin{array}{l}17.05 \pm 4.59 \\
23.04 \pm 8.93\end{array}$ & $\begin{array}{l}19.73 \pm 7.77 \\
14.42 \pm 4.37\end{array}$ & $\begin{array}{l}0.26 \\
0.002^{\bullet}\end{array}$ \\
\hline$P$ value & & 0.09 & $0.02 *$ & \\
\hline
\end{tabular}

Notes: Values are Mean \pm SD. ${ }^{*} \mathrm{P}<0.05$ in paired-sample $t$-test vs before exhaustive exercise. ${ }^{\bullet} \mathrm{P}<0.05$ in independent-sample $t$-test between two groups 
participation in the synthesis of glutathione. Glutathione system has been shown as one of the main mechanisms for oxidative stress reduction. ${ }^{29}$ Intense exercise has been reported to influence glutathione synthesis and utilization. In fact, intense exercise results in glutathione depletion and muscle damage. ${ }^{30,31}$ Moreover, reduced glutathione and increased oxidative stress in intense exercise has been revealed to be followed by lowering serum glutamine levels. ${ }^{32}$ Therefore, as shown in the present study, glutamine supplementation can be effective in increasing glutathione levels after an exhausting exercise and prevents from sport damage.

In our study, glutamine supplementation increased TAC serum levels and subsequently reduced serum levels of MDA. Determination of serum levels of TAC and MDA is one of the most important markers of oxidative stress. MDA is a principal indicator of oxidative stress and increasing in its serum level indicates an increase in oxidative stress. ${ }^{33}$ Furthermore, TAC plays an important role against oxidative stress and reduction of cellular damage caused by it. ${ }^{34}$ Our results are consistent with previous findings that reported the glutamine supplementation increased total antioxidant capacity of the body and reduced oxidative stress. ${ }^{35,36}$ Also, it has been shown that the administration of 3 months of aerobic exercise training has been improved systemic oxidative stress and inflammatory biomarkers in individuals with metabolic syndrome. ${ }^{37}$ Moreover, glutamine consumption before intense and prolonged physical activity has been reported to prevent oxidative stress caused by the activity. ${ }^{14}$ Also, Zang et al demonstrated that glutamine intake can reduce oxidative stress by increasing the antioxidant capacity of the body. ${ }^{38}$ Thus, the increase in the TAC serum levels and the decrease in the MDA can result in decreasing the oxidative stress and compounds such as glutamine having this property can be useful in controlling stressful conditions such as exhaustive exercise.

One of the most interesting results of our study was the reduction of serum levels of hs-CRP after supplementation with glutamine and also after exhaustive exercise. Studies have indicated that hs-CRP is a sensitive marker of inflammation and its serum level decreases by improving the inflammatory process. ${ }^{39}$ In the strenuous exercise, increased levels of oxidative stress and inflammatory markers have been observed and antioxidant effects of glutamine may be effective in reducing the inflammatory process. ${ }^{40}$ However, glutamine has been found to inhibit inflammatory processes by inhibiting the NF- $\mathrm{BB}$ pathway. ${ }^{41}$ In fact, intense exercise results in substantially increasing in mitochondrial electron flux followed by ROS production and oxidative stress. ROS stimulate inflammation through NF- $\mathrm{BB}$ and STAT pathways. ${ }^{42}$ Also, ROS stimulate the production of inflammatory markers such as hs-CRP. Increased serum levels of hs-CRP observed after exhaustive exercise in our study is in line with findings of the study by Vincent et al. ${ }^{43}$ Inflammatory reaction in intensive exercise has been reported as one of the reasons for sport injury. ${ }^{44}$ Glutamine may play a role in improving and controlling inflammatory processes. ${ }^{45}$ Thus, in this study, the reduction of the inflammatory process as a result of glutamine supplementation is considered a very important finding because it can prevent sport damage associated with increased inflammation in sport and glutamine consumption can be beneficial for young healthy males performing intense physical activities.

In our study, exercise had no significant effect on serum levels of MMP2 and MMP9. Similarly, glutamine supplementation had no effect on serum levels of MMP2 and MMP9 after supplementation and an exhaustive exercise. Modifications in serum levels of MMP2 and MMP9 can be valuable factors to assess the effect of exercise on inflammatory condition. ${ }^{46}$ However, there are limited studies about the effects of training on the serum alterations in MMP2 and MMP9. Some studies have indicated that serum levels of MMP2 and MMP9 are changed after exercise. For example, Giganti et $\mathrm{al}^{15}$ in a cohort study indicated that various physical activities regulate levels of circulating MMP2 and -9 in breast cancer survivors, and Carmeli et al revealed that high-intensity exercise involving treadmill running for 2 weeks increased levels of MMP2 in skeletal muscles in rats and did not alter MMP9 levels in the muscles. ${ }^{47}$ Urso et al showed an increase in MMP9 serum levels after an acute resistance exercise test following 8 weeks of exercise training but no change was observed in serum levels of MMP $2 .{ }^{48}$ Also, some studies have shown that MMP2 and MMP9 are the major MMPs involving in the exercise studies and their levels have been found to reach peak values within a relatively short time following a single intense exercise. ${ }^{46}$ However, Mackey et al consistent with our findings showed that after two exercise bouts, a $10-\mathrm{km}$ road running and a deep-water running session, serum levels of MMP2 and MMP9 remained unchanged. ${ }^{49}$ Therefore, serum levels of MMP2 and MMP9 may be influenced by the duration of training such as chronic and acute exercise. Also, changes in serum levels of MMP2 and MMP9 are probably related to different dose-response factors of physical activities such as type, intensity, volume, and frequency. ${ }^{46}$ These findings suggest 
that the mode of exercise and duration of exercise may influence MMP response to exercise. However it should be said that different methods used for the determination of MMP2 and MMP9 levels such as muscle biopsies and plasma concentrations can influence the evaluation of matrix metalloproteinases. ${ }^{46}$ Hence, further studies are required to completely explain the connection between the role of exercise in the MMP2 and MMP9 response.

Also, considering limited evidence available about the effects of glutamine supplementation on serum levels of MMP2 and MMP9, our study is the first study showed the effect of glutamine supplementation on serum levels of MMP9 and MMP2. However, some studies have shown the effect of other antioxidant compounds on serum levels of MMP2 and MMP9. In this regard, recent studies have shown that antioxidant compounds such as $\alpha$-lipoic acid, resveratrol, quercetin and CLA (conjugated linoleic acid) can reduce levels of matrix metalloproteinase. ${ }^{50-53}$ In addition, regular intake of pomegranate juice as an antioxidant during 2 weeks of intervention has been reported to significantly decrease MMP2 and MMP9 in young healthy males. ${ }^{10}$ In these studies, the reduction of MMP2 and MMP9 has mainly been related to the antiinflammatory effects of the above-mentioned compounds. However, anti-inflammatory effects of glutamine have been confirmed in numerous studies..$^{11,53,54,55}$ In our study, glutamine supplementation did not influence serum levels of MMP2 and MMP9. The ineffectiveness of this supplementation on serum levels of MMP2 and MMP9 can be due to several factors such as not using higher doses of glutamine or a short period of intervention. It seems that glutamine supplementation in a short period of time has less effect or no effect on MMP2 and MMP9 after an exhaustive exercise. There were some limitations in this study including the measurement of serum glutamine and the absence of proper cooperation on the part of some participants during the study.

\section{Conclusion}

Results of this study showed that some biochemical factors are time-dependent and can increase or decrease over time, and also serum levels of hs-CRP and MDA decreased with glutamine supplementation along with the increase in the TAC serum levels, but this supplementation had no effect on serum levels of MMP2 and MMP9 after an exhaustive exercise.

\section{Data Sharing Statement}

The individual participant's data underlying published results reported in this study can be accessed with approval from the corresponding author after 6 months of publication of the main results. The study protocol, statistical analysis plan, and clinical study report will also be available.

\section{Acknowledgements}

The authors are grateful to Ardabil University of Medical Sciences for financial support as well as to all the participants.

\section{Disclosure}

The authors report no conflict of interest.

\section{References}

1. Naghibi S. The effect of short-term creatine supplementation on cardiovascular damage markers after an exhaustive exercise session in elite karate athletes. Sport Physiol. 2014;6(22):15-28.

2. Nemes R, Koltai E, Taylor A, Suzuki K, Gyori F, Radak Z. Reactive oxygen and nitrogen species regulate key metabolic, anabolic, and catabolic pathways in skeletal muscle. Antioxidants. 2018;7(7):1-13. doi:10.3390/antiox7070085

3. Nieman DC, Davis J, Brown VA, et al. Influence of carbohydrate ingestion on immune changes after $2 \mathrm{~h}$ of intensive resistance training. $J \mathrm{Appl}$ Physiol. 2004;96(4):1292-1298. doi:10.1152/japplphysiol.01064.2003

4. Maquoi E, Peyrollier K, Agnes N, Foidart J-M, Frankenne F. Regulation of membrane-type 1 matrix metalloproteinase activity by vacuolar $\mathrm{H}+--$ ATPases. Biochem J. 2003;373(1):19-24. doi:10.1042/bj20030170

5. Kawasaki H, Manickam A, Shahin R, Ote M, Iwanaga M. Expression of matrix metalloproteinase genes during basement membrane degradation in the metamorphosis of Bombyx mori. Gene. 2018;638:26-35. doi:10.1016/j.gene.2017.09.031

6. Haas T, Milkiewicz M, Davis S, et al. Matrix metalloproteinase activity is required for activity-induced angiogenesis in rat skeletal muscle. Am J Physiol Heart Circ Physiol. 2000;279(4):H1540-H7. doi:10.1152/ajpheart.2000.279.4.H1540

7. Amirsasan R, Mirshafiei A, Gaeini A, et al. Effects of exhaustive aerobic exercise on matrix metaloproteases activity in athletes and non-athletes. World J Sport Sci. 2011;4:185-191.

8. Choi Y-C, Dalakas MC. Expression of matrix metalloproteinases in the muscle of patients with inflammatory myopathies. Neurology. 2000;54(1):65-71. doi:10.1212/WNL.54.1.65

9. Yavari A, Javadi M, Mirmiran P, Bahadoran Z. Exercise-induced oxidative stress and dietary antioxidants. Asian J Sports Med. 2015;6(1):1-7. doi:10.5812/asjsm.24898

10. Mazani M, Fard AS, Baghi AN, Nemati A, Mogadam RA. Effect of pomegranate juice supplementation on matrix metalloproteinases 2 and 9 following exhaustive exercise in young healthy males. J Pak Med Assoc. 2014;64:785-790.

11. Raizel R, Leite JSM, Hypólito TM, et al. Determination of the anti-inflammatory and cytoprotective effects of 1-glutamine and 1-alanine, or dipeptide, supplementation in rats submitted to resistance exercise. $B r \quad J$ Nutr. 2016;116(3):470-479. doi:10.1017/ S0007114516001999

12. Castell LM. Glutamine supplementation in vitro and in vivo, in exercise and in immunodepression. Sports Med. 2003;33(5):323-345. doi:10.2165/ 00007256-200333050-00001

13. Cruzat VF, Rogero MM, Tirapegui J. Effects of supplementation with free glutamine and the dipeptide alanyl-glutamine on parameters of muscle damage and inflammation in rats submitted to prolonged exercise. Cell Biochem Funct. 2010;28(1):24-30. doi:10.1002/cbf.v28:1 
14. Nakhostin-Roohi B, Javanamani R. The effect of glutamine supplementation on exercise-induced oxidative stress. J Adv Agri Technol. 2015;2(1):8-12. doi:10.12720/joaat.2.1

15. Giganti MG, Tresoldi I, Sorge R, et al. Physical exercise modulates the level of serum MMP-2 and MMP-9 in patients with breast cancer. Oncol Lett. 2016;12(3):2119-2126. doi:10.3892/ol.2016.4887

16. Machin D, Campbell MJ, Tan SB, Tan SH. Sample Sizes for Clinical, Laboratory and Epidemiology Studies. Vol. 22. Wiley Online Library; 2018:361-362.

17. Wernerman J. Glutamine supplementation. Ann Intensive Care. 2011;1(1):1-6. doi:10.1186/2110-5820-1-25

18. Gleeson M. Dosing and efficacy of glutamine supplementation in human exercise and sport training. J Nutr. 2008;138(10):2045S-9S. doi:10.1093/jn/138.10.2045S

19. Kim YJ, Chun H, Kim C-H. Exaggerated response of systolic blood pressure to cycle ergometer. Ann Rehabil Med. 2013;37(3):364-372. doi:10.5535/arm.2013.37.3.364

20. Lahart IM, Carmichael AR, Nevill AM, Kitas GD, Metsios GS. The effects of a home-based physical activity intervention on cardiorespiratory fitness in breast cancer survivors; a randomised controlled trial. J Sports Sci. 2018;36(10):1077-1086. doi:10.1080/02640414.2017.1356025

21. Dlugosz EM, Chappell MA, Meek TH, et al. Phylogenetic analysis of mammalian maximal oxygen consumption during exercise. $J$ Exp Biol. 2013;216(24):4712-4721. doi:10.1242/jeb.088914

22. Pollock ML, Foster C, Schmidt D, Hellman C, Linnerud A, Ward A. Comparative analysis of physiologic responses to three different maximal graded exercise test protocols in healthy women. Am Heart J. 1982;103(3):363-373. doi:10.1016/0002-8703(82) 90275-7

23. Li H, Miao W, Ma J, et al. Acute exercise-induced mitochondrial stress triggers an inflammatory response in the myocardium via NLRP3 inflammasome activation with mitophagy. Oxid Med Cell Longev. 2016;2016:1-11.

24. Bessa AL, Oliveira VN, Agostini GG, et al. Exercise intensity and recovery: biomarkers of injury, inflammation, and oxidative stress. J Strength Cond Res. 2016;30(2):311-319. doi:10.1519/JSC.0b013e31828flee9

25. Clifford T, Bell O, West DJ, Howatson G, Stevenson EJ. The effects of beetroot juice supplementation on indices of muscle damage following eccentric exercise. Eur J Appl Physiol. 2016;116 (2):353-362. doi:10.1007/s00421-015-3290-x

26. Cruzat VF, Krause M, Newsholme P. Amino acid supplementation and impact on immune function in the context of exercise. J Int Soc Sports Nutr. 2014;11(1):1-13. doi:10.1186/s12970-014-0061-8

27. Moura CS, Lollo PCB, Morato PN, Risso EM, Amaya-Farfan J. Modulatory effects of arginine, glutamine and branched-chain amino acids on heat shock proteins, immunity and antioxidant response in exercised rats. Food Funct. 2017;8(9):3228-3238. doi:10.1039/ C7FO00465F

28. Szpetnar M, Luchowska-Kocot D, Boguszewska-Czubara A, Kurzepa J. The influence of manganese and glutamine intake on antioxidants and neurotransmitter amino acids levels in rats' brain. Neurochem Res. 2016;41(8):2129-2139. doi:10.1007/s11064-0161928-7

29. Petry ER, Cruzat VF, Heck TG, de Bittencourt PIH Jr, Tirapegui J. L-Glutamine supplementations enhance liver glutamine-glutathione axis and heat shock factor-1 expression in endurance-exercise trained rats. Int J Sport Nutr Exerc Metab. 2015;25(2):188-197. doi:10.1123/ijsnem.2014-0131

30. Aoi W, Ogaya Y, Takami M, et al. Glutathione supplementation suppresses muscle fatigue induced by prolonged exercise via improved aerobic metabolism. J Int Soc Sports Nutr. 2015;12 (1):1-8. doi:10.1186/s12970-015-0067-x

31. Gomez-Cabrera MC, Borrás C, Pallardó FV, Sastre J, Ji LL, Viña J. Decreasing xanthine oxidase-mediated oxidative stress prevents useful cellular adaptations to exercise in rats. J Physiol. 2005;567 (1):113-120. doi:10.1113/jphysiol.2004.080564
32. Agostini F, Biolo G. Effect of physical activity on glutamine metabolism. Curr Opin Clin Nutr Metab Care. 2010;13(1):58-64. doi:10.1097/MCO.0b013e328332f946

33. Sarban S, Kocyigit A, Yazar M, Isikan UE. Plasma total antioxidant capacity, lipid peroxidation, and erythrocyte antioxidant enzyme activities in patients with rheumatoid arthritis and osteoarthritis. Clin Biochem. 2005;38(11):981-986. doi:10.1016/j.clinbiochem.2005.08.003

34. El-Shenawy NS, Hamza RZ, Ismail H, Khaled HE. Efficacy of $\alpha-$ lipoic acid against oxidative stress and histopathological changes induced by dimethylnitrosamine in liver male mice. Exp Toxicol Pathol. 2016;6(3):102-112.

35. Stangl R, Szijarto A, Onody $P$, et al. Reduction of liver ischemia-reperfusion injury via glutamine pretreatment. J Surg Res. 2011;166(1):95-103. doi:10.1016/j.jss.2009.09.047

36. Lin Z, Cai F, Lin N, Ye J, Zheng Q, Ding G. Effects of glutamine on oxidative stress and nuclear factor- $\kappa \mathrm{B}$ expression in the livers of rats with nonalcoholic fatty liver disease. Exp Ther Med. 2014;7 (2):365-370. doi:10.3892/etm.2013.1434

37. Farinha JB, Steckling FM, Stefanello ST, et al. Response of oxidative stress and inflammatory biomarkers to a 12-week aerobic exercise training in women with metabolic syndrome. Sports Med Open. 2015;1(1):1-10. doi:10.1186/s40798-015-0011-2

38. Zhang W-X, Zhou L-F, Zhang L, et al. Protective effects of glutamine preconditioning on ischemia-reperfusion injury in rats. Hepatobiliary Pancreat Dis Int. 2011;10(1):78-82. doi:10.1016/S1499-3872(11) 60011-8

39. Kim JH, Doo SW, Yang WJ, Song YS, Kwon -S-S. Association between high-sensitivity $\mathrm{C}$-reactive protein and lower urinary tract symptoms in healthy Korean populations. Urology. 2015;86 (1):139-144. doi:10.1016/j.urology.2015.03.035

40. Habibi Neghad A, Seiavoshy H, Samavatisharif M. The Effect of an exhaustive exercise and glutamine supplementation on LDH, CPK and CPR indexes in non-athlete women students. Bull Env Pharmacol Life Sci. 2015;4(6):1-6.

41. Kretzmann NA, Fillmann H, Mauriz JL, et al. Effects of glutamine on proinflammatory gene expression and activation of nuclear factor kappa B and signal transducers and activators of transcription in TNBS-induced colitis. Inflamm Bowel Dis. 2008;14(11):1504-1513. doi:10.1002/ibd.20543

42. Ji L, Gomez-Cabrera M, Steinhafel N, Vina J. Acute exercise activates nuclear factor (NF)- $\mathrm{BB}$ signaling pathway in rat skeletal muscle. FASEB J. 2004;18(13):1499-1506. doi:10.1096/fj.04-1846com

43. Vincent HK, Bourguignon CM, Vincent KR, Weltman AL, Bryant M, Taylor AG. Antioxidant supplementation lowers exercise-induced oxidative stress in young overweight adults. Obesity. 2006;14 (12):2224-2235. doi:10.1038/oby.2006.261

44. Leeder J, Gissane C, van Someren K, Gregson W, Howatson G. Cold water immersion and recovery from strenuous exercise: a meta-analysis. Br J Sports Med. 2012;46(4):233-240. doi:10.1136/ bjsports-2011-090061

45. Kang K, Shu X-L, Zhang Y-S, Liu X-L ZJ. Effect of glutamine enriched nutrition support on surgical patients with gastrointestinal tumor: a meta-analysis of randomized controlled trials. Chin Med J. 2015;128(2):245-251. doi:10.4103/0366-6999.149219

46. Da Cunha Nascimento D, Durigan R, Tibana RA, Durigan JLQ, Navalta JW, Prestes J. The response of matrix metalloproteinase-9 and-2 to exercise. Sports Med. 2015;45(2):269-278. doi:10.1007/ s40279-014-0265-8

47. Carmeli E, Moas M, Lennon S, Powers SK. High intensity exercise increases expression of matrix metalloproteinases in fast skeletal muscle fibres. Exp Physiol. 2005;90(4):613-619. doi:10.1113/ expphysiol.2004.029462

48. Urso ML, Pierce JR, Alemany JA, Harman EA, Nindl BC. Effects of exercise training on the matrix metalloprotease response to acute exercise. Eur J Appl Physiol. 2009;106(5):655-663. doi:10.1007/ s00421-009-1063-0 
49. Mackey AL, Donnelly AE, Swanton A, Murray F, TurpeenniemiHujanen T. The effects of impact and non-impact exercise on circulating markers of collagen remodelling in humans. J Sports Sci. 2006;24(8):843-848. doi:10.1080/02640410500231470

50. Lee HS, Na MH, Kim WK. $\alpha$-Lipoic acid reduces matrix metalloproteinase activity in MDA-MB-231 human breast cancer cells. Nutr Res. 2010;30(6):403-409. doi:10.1016/j.nutres.2010.06.009

51. Pereira SC, Parente JM, Belo VA, et al. Quercetin decreases the activity of matrix metalloproteinase-2 and ameliorates vascular remodeling in renovascular hypertension. Atherosclerosis. 2018;270:146-153. doi:10.1016/j. atherosclerosis.2018.01.031

52. Chen Z, Hu L, Lu M, Shen Z. Resveratrol reduces matrix metalloproteinases and alleviates intrahepatic cholestasis of pregnancy in rats. Can J Physiol Pharmacol. 2015;94(4):402-407. doi:10.1139/cjpp-2015-0454
53. Matin S, Nemati A, Ghobadi H, Alipanah-Moghadam R, Rezagholizadeh L. The effect of conjugated linoleic acid on oxidative stress and matrix metalloproteinases 2 and 9 in patients with COPD. Int J Chron Obstruct Pulmon Dis. 2018;13:1449-1454. doi:10.2147/ COPD.S155985

54. Jain P, Khanna N. Evaluation of anti-inflammatory and analgesic properties ofl-glutamine. Agents Actions. 1981;11(3):243-249. doi:10.1007/ BF01967621

55. Shih Y-M, Shih J-M, Pai M-H, Hou Y-C, Yeh C-L, Yeh S-L. Glutamine administration after sublethal lower limb ischemia reduces inflammatory reaction and offers organ protection in ischemia/reperfusion injury. J Parenter Enteral Nutr. 2016;40(8):1122-1130. doi:10.1177/0148607115587949

\section{Publish your work in this journal}

Drug Design, Development and Therapy is an international, peerreviewed open-access journal that spans the spectrum of drug design and development through to clinical applications. Clinical outcomes, patient safety, and programs for the development and effective, safe, and sustained use of medicines are a feature of the journal, which has also been accepted for indexing on PubMed Central. The manuscript management system is completely online and includes a very quick and fair peer-review system, which is all easy to use. Visit http://www. dovepress.com/testimonials.php to read real quotes from published authors. 